\title{
THE CONCEPT OF NEGATION
}

\begin{abstract}
Using Evidence Logic (EL), a logic which is conceptually to Classical Logic and which is equipped with both gradational confirmatory predications and gradational refutatory predications, further investigation of some of the persistent problematic aspects of negation is facilitated. This perspective helps to illuminate distinction of "absence of evidence", and may help further understanding of the semantics of negation.
\end{abstract}

\section{CONTENTS}

1. Introduction, p. 36

2. Basing semantics on evidence, p. 36

3. Evidence logic, p. 38

4. The concept of negation, p. 39

5. Discussion, p. 44

Received April 2, 1997. Revised February 12, 1998 


\section{Introduction}

A number of nonclassical systems of logic are currently being generated in attempts to address the knowledge representation and knowledge processing areas of Artificial Intelligence (AI). In this process a number of philosophical issues, especially in regard to the concept of negation, though foundationally important to the work being attempted, remain in need of distillation and analysis. In this paper, making use of a new logic called Evidence Logic (EL) which is conceptually antecedent to Classical Logic (CL), we will attempt to contribute to philosophical discussions aimed toward addressing this need.

It should perhaps be made clear at the outset that, although we shall in the course of the paper consider a number of aspects of the concept of negation, in a sense which will be made clear in the context of this paper a basic characteristic of negation $(\neg)$ which will be assumed throughout is the following. For any sentence $\varphi, " \neg \varphi$ " means "it is not the case that $\varphi$ "; hence, for us $\neg \neg \varphi$ holds if and only if $\varphi$ holds.

In Section 2 we will explicate the semantic level using which we shall proceed with our analysis of the concept of negation, and discuss the reasonableness and utility of this perspective. In Section 3 we will present EL in sufficient detail for the needs at hand. In Section 4 EL will be used to analyze some of the philosophical problems surrounding negation, referencing along the way (1) some of the many philosophers, beginning with Aristotle, who have wrestled valiantly with the difficulties surrounding negation and (2) some of the current insights about negation which are being provided by workers in AI. In Section 5, we will discuss how our analysis, in spite of the rather shallow semantic level assumed, may help in shedding some light on the deeper issues involving negation.

\section{Basing semantics on evidence}

The ultimate nature of reality continues to elude us, and that situation may persist for a long time to come. Yet we do represent and process a myriad of approximative models of reality, tools with which we analyze, manipulate, and, yes, even come to understand better the universe about us. Honesty alone forces us to admit that, since the ultimate nature of reality remains 
yet unknown, it is with evidence regarding that reality that we are in fact dealing in our scientific endeavors. This evidence is indicative in nature, and is built upon a great multitude and variety of data which variously combine to provide confirmatory and refutatory "atomic evidence" in models about the nature of matter and phenomena. (See also [Faust 1990, 1992].)

For a long time (say, the last 30,000 years) we have very successfully come to understand our physical universe well enough to build a wide variety of tools for the automation and extension of our physical abilities: the wheel, pulleys, the construction crane, the airplane, .... In contrast, automation and extension of our mental abilities, throughout this long history, has been rather feeble: arithmetic, algebra, physics, and the like, are in fact not even usually thought of in terms of their automation function at all since traditionally it has been we ourselves who have provided the mental engine used in running these tools. But now we are surely at the dawn of a new age, the computer age: the electronic computer and its descendants will provide an engine sufficient for increasingly efficacious automation and extension of our mental abilities.

AI is one of the sciences of this automation and extension of our mental abilities. (Further, it might be noted, AI also includes work, such as robotics, which seeks to integrate its automation and extension of mental abilities with the traditional automation and extension of physical abilities, thus making AI also a science working toward the automation and extension of the combined physical and mental abilities of humans.) AI is beginning to turn its attention to the possibility of building AI systems which will represent and process evidence in a variety of complex domains. In many of these domains the evidence comes in degrees rather than being absolute. For example, in a process control environment it may well be the case that evidence is assigned a degree of certainty based upon input from many sensors which each contribute variously to the degree of certainty of the evidence; further, faults intrinsic to the sensors, or inherent in the environment, may also affect the degree of certainty of the evidence. For instance, the momentary absence of a reading from one of the sensors may cause a decrease in the certainty levels assigned to any atomic evidence whose ascertainment makes use of that sensor. Further, conclusions to be drawn in such domains of evidence, whether the evidence is absolute or not, will sometimes have a degree of uncertainty to them. Such less than certain conclusions may thrust themselves upon us by necessity, because of uncertain evidence or because a real time processing environment precludes the luxury of complete argumentation to a crisp conclusion. Or such conclusions may in fact be preferred, 
for example because crisp conclusions are not required for the triggering of appropriate action.

Our perspective here, then, sets out evidence as the ontological base of our semantics. And, it should be emphasized, we are not thereby positing any assertion of 'correctness' of this view; we simply find this model widely and successfully used, for example in AI as discussed above, and seek a reasonable foundation for it. There are, of course, a number of eventualities which may attain with regard to the ultimate nature of reality, and we shall digress briefly, as an argument in favor of our perspective of an ontology of evidence, to consider some of them.

We may never come to completely understand the nature of reality, in which case an ontology composed of evidence will certainly be worthwhile. Alternatively, we may indeed at some future time come to completely understand the nature of reality. It may be found, however, that this complete understanding may not be workably representable, in which case an ontology of evidence will again be useful. Alternatively, it may indeed be workably representable. If, in spite of the fact that it is workably representable, it is not processible (for example, because of limitations imposed by an inherent complexity of our representation of our knowledge of the nature of reality or because of an inherent complexity of processing of that knowledge), then again an ontology of evidence will prove useful. Alternatively, it may be processible. If it is two-valued, then in the long interim of probably thousands of years before this completely understood workably representable processible knowledge of the nature of reality emerges yet again an ontology of evidence will be useful for us. If, finally, it is not two-valued, then certainly our having worked with an ontology of evidence will have provided us with research experiences which will be helpful to us in constructing efficacious representational frameworks for handling that completely understood workably representable processible many valued reality. So every branch in this tree of possibilities, at least that part of the tree we have briefly considered in this digression, leads to a substantial utility of one sort or another for logics based on an ontology of evidence.

\section{Evidence logic (EL)}

EL as it currently exists [Faust 1993, 1994] is a first-order predicate logic with equality and a finite number of finitary function and relation symbols, where, relative to an Evidence Space $\mathrm{E}_{n}=\{i /(n-1): i=1, \ldots, n-1\}$ 
(for any fixed $n \geqslant 2$ ) of evidence annotations, with smallest evidence value $\varepsilon=1 /(n-1)$, there are associated with any $k$-ary relation symbol $R$, any terms $t_{1}, \ldots, t_{k}$, and any evidence value $e$ in $\mathrm{E}_{n}$ the following (respectively) confirmatory and refutatory atomic formulas:

$$
R_{c} t_{1} \ldots t_{k}: e \text { and } R_{r} t_{1} \ldots t_{k}: e .
$$

That is, in EL atomic formulas are either confirmatory or refutatory, and are evidential. Further, the logical axioms of EL consist of any usual set of logical axioms plus additional axioms which assure that 'stronger evidence strictly entails weaker evidence'. With regard to the semantics of EL, models $\mathcal{A}$ of EL are similarly equipped, as motivated in section 2 above, providing evidentially annotated confirmatory and refutatory relations $R_{c}^{\mathcal{A}}$ and $R_{r}^{\mathcal{A}}$ interpreting $R_{c}$ and $R_{r}$ (respectively) for each relation symbol $R$.

We will refer to any Evidence Logic with Evidence Space $\mathrm{E}_{n}$ as $\mathrm{EL}_{n}$. Trivializing the refutatory predicates in $\mathrm{EL}_{n}$ yields the Confirmatory Evidence Logic $\mathrm{CEL}_{n}$ while specializing to the Evidence Space $\mathrm{E}_{2}=\{1\}$ yields the "absolute" Evidence Logic $\mathrm{EL}_{2}$. Note that $\mathrm{EL}_{2}$ is much like Classical Logic except that $\mathrm{EL}_{2}$ provides for refutatory atomic assertions in addition to confirmatory ones whereas Classical Logic provides only the latter; this distinguishing character of $\mathrm{EL}_{2}$ will play an important roll in our explication of the concept of negation in Section 4. Finally, note that $\mathrm{CEL}_{2}$ is both confirmatory and absolute, and is indeed precisely Classical Logic.

It will suffice in this paper, with its focus on the concept of negation, to consider only the propositional fragment of EL, containing, let us say, the proposition symbol $\mathrm{P}$. With regard to the Evidence Space $\mathrm{E}_{n}$, the reader may find it a convenient concession to the decimal mind to think, at the level of an example, in terms of $\mathrm{E}_{11}=\{.1, .2, \ldots, .9,1\}$ in which case $\varepsilon=.1$. In practice, $n$ is, within constraints imposed by the implementation environment, determined by the granularity of the evidence.

\section{The concept of negation}

The algebraization of logic which progressed substantially beginning in the mid-nineteenth century, along with the decisive influence of Frege, rather firmly fixed a standard characterization of predicate logic, and in particular negation, which has persisted throughout the twentieth century. Under this characterization: (1) negation is sentential negation, (2) atomic information is only confirmatory in character, and (3) atomic information is absolute. 
With regard to (1) we shall raise no disagreement, and in fact we will make reference to the main traditional alternative to sentential negation, namely term negation, in a manner which in no way is meant to suggest that term negation be seen as a serious competitor to the standard characterization of negation as sentential negation. However, with regard to (2) and (3) we shall argue that it is both reasonable and productive that a fundamental first-order predicate logic should be developed, herein we suggest along the lines of Evidence Logic, which $\left(2^{\prime}\right)$ is equipped to handle atomic information which is confirmatory and also atomic information which is refutatory and $\left(3^{\prime}\right)$ is equipped to handle atomic information which is less than certain.

Let us first compare the nature of negation in CL and $\mathrm{EL}_{2}$. In CL to assert $\mathrm{P}$ is to assert that there is absolute confirmatory evidence with respect to $\mathrm{P}$, while to assert $\neg \mathrm{P}$ is to assert that it is not the case that there is absolute confirmatory evidence with respect to $\mathrm{P}$. Is the latter claim equivalent to the assertion that there is absolute refutatory evidence with respect to $\mathrm{P}$ ? This matter is just not spoken to in CL, since it contains no machinery concerning the refutatory. In fact, the reader may well be saying, in answer to this question: "Of course not ... but, anyhow, it is simply part of the deliberate construction of CL that it contains only machinery for handling the confirmatory." Agreed. But, especially in view of Section 2 above, it is not unreasonable that a fundamental first-order predicate logic be equipped with machinery for handling both the confirmatory and the refutatory. This is exactly what one finds in $\mathrm{EL}_{2}$. In $\mathrm{EL}_{2}$ to assert $\mathrm{P}_{c}$ (we will uniformly omit the evidence level, which is always 1 , in $\mathrm{EL}_{2}$ ) is to assert that there is absolute confirmatory evidence with respect to $\mathrm{P}$, while to assert $\neg \mathrm{P}_{c}$ is to assert that it is not the case that there is absolute confirmatory evidence with respect to $\mathrm{P}$. And to assert $\mathrm{P}_{r}$ is to assert that there is absolute refutatory evidence with respect to $\mathrm{P}$, while to assert $\neg \mathrm{P}_{r}$ is to assert that it is not the case that there is absolute refutatory evidence with respect to $\mathrm{P}$.

Turning now to the more general case of $\mathrm{EL}_{n}$ we find that $\mathrm{EL}_{n}$ has not only the above advantage over CL but also the further advantage of the evidence gradations provided by the Evidence Space $\mathrm{E}_{n}$, annotatively a part of the atomic formulas of $\mathrm{EL}_{n}$. Let $e$ be any one of the evidence values in $\mathrm{E}_{n}$. To assert $\mathrm{P}_{c}: e$ is to assert that there is confirmatory evidence at level $e$ regarding $\mathrm{P}$, while to assert $\neg \mathrm{P}_{c}: e$ is to assert that it is not the case that there is confirmatory evidence at level $e$ regarding $\mathrm{P}$. And to assert $\mathrm{P}_{r}: e$ is to assert that there is refutatory evidence at level $e$ regarding $\mathrm{P}$, while to assert $\neg \mathrm{P}_{r}: e$ is to assert that it is not the case that there is refutatory evidence at level $e$ regarding $\mathrm{P}$. 
The distinctions, for example between $\neg \mathrm{P}_{c}: e$ and $\mathrm{P}_{r}: e$, which are made so evident here in EL, and which cannot be made in CL, are important ones, as has been demonstrated by recent and current work in AI (cf. Section 2 above). Further, CL is easily seen to be an axiomatizable extension of EL, and hence indeed EL is conceptually antecedent to CL. It is from this perspective that EL is recommended as an example of a reasonable and productive replacement of CL as a fundamental first-order predicate logic. Finally, the interested reader is encouraged to consult [Faust 1993], where EL is characterized and it is proven in exactly what sense EL generalizes CL, in terms of Boolean algebras of sentences and topological spaces of models.

Let us turn now briefly to the historical evolution of the concept of negation. There have been a number of workers in logic throughout history who have carefully considered the concept of negation, who have tried valiantly to bring clarity to the substantial philosophical problems surrounding negation. Through the peripatetics, the stoics, the nyaya-sutra and navya-nyaya of India, the medieval logicians and beyond, right up through the classical and nonclassical logicians of the twentieth century, we find a steady march of logicians and philosophers of logic who raise and grapple with the problems surrounding negation. While recommending to the interested reader the study of the vast historical literature which makes this clear, let us here consider only two examples of this remarkable phenomenon: Aristotle in his Prior Analytics and the 'doctrine of negation' of the navya-nyaya tradition of India. In both cases we find able logicians facing squarely the problem that negation as "it is not the case that" seems to be a correct explication of that part of the concept of negation having to do with 'absence', but that somehow that part of the concept of negation having to do with 'otherness' remains unexplicated. That is, classical negation is certainly part of the story, but in some sense it fails to provide tools adequate to the explication of the relation between the absence of the confirmatory and the refutatory.

Consider the following from Aristotle's Prior Analytics, Book I, Chapter XLVI [Aristotle]:

It makes no little difference in establishing or refuting a proposition whether we suppose that 'not to be so-and-so' and 'to be not-so-and-so' mean the same or something different: e.g. whether 'not to be white' means the same as 'to be not-white'. For it does not mean the same; the negation of 'to be white' is not 'to be not-white' but 'not to be white'.

And again, later in the same chapter, we find the following:

Let A stand for 'to be good', B for 'not to be good', C for 'to be not-good' and D for 'not to be not-good'. Then either A or B will apply 
to everything, but they can never both apply to the same subject; and either C or D will apply to everything, but they can never both apply to the same subject. Also B must apply to everything to which C applies. For if it is true to say 'it is not-white', it is also true to say 'it is not white'; since it is impossible that a thing should at the same time be white and not-white $[\ldots]$.

Also, let us consider the navya-nyaya tradition of India. We quote from [Matilal, p. 90]:

The Naiyayikas [...] argue that if bread's presence can lead one to construe bread as a property (dharma) of the table, bread's absence may alike lead us to construe the bread-absence as a property of the table.

And in Sharma's translation of the Apohasiddhih by Ratnakirti we find the following [Sharma, p. 51-55]:

That which is envisaged as a positive entity also entails the exclusion of what is other, and this is called [...] differentiation [...]. By the term differentiation what is intended is not merely a positive entity A; nor merely the exclusion of non-A; [rather] the meaning of the term is the positive [entity] A qualified by the exclusion of non-A. [...] Hence, the statement 'understanding of the word cow' is said to mean 'the understanding of the distinct positive entity qualified by the exclusion of all that is non-cow'. [...] The term 'cow' indeed [includes] the negation of 'non-cow'. [...] Otherwise [...] one could well tie [up] horses etc. when asked to tie up a cow!

And finally, from [Sharma, p. 95]:

Thus a positive statement comprises the particular qualified by the negation of the other and discriminated from the class of dissimilar instances.

It is not our purpose here to critique the work of earlier logicians such as that indicated in the above. There has been considerable research done in that direction already, though certainly much remains to be done. Rather, we cite the above in the present context in order to emphasize the fact that throughout history logicians have returned again and again to the problem of negation, attempting to bring clarity to the many issues involved. Let us attempt to look beyond the technicalities of how these earlier logicians attempted to analyze and resolve their perplexities. Let us, that is, simply attempt to paint in very broad strokes what, in the main, these perplexities themselves were, at least for Aristotle and Ratnakirti. 
It seems clear that they both acknowledge propositional negation, that is, negation in the sense of "it is not the case that". However, it seems equally clear that neither of them is satisfied that this is all that needs to be said about negation. In a manner not unlike many others in the long history of logic, they go on at length analyzing and systematizing, in regard to other dimensions of the concept of negation which are not addressed by propositional negation. Propositional negation addresses that part of the concept of negation having to do with absence; that is, the negation of a proposition is associated with THE ABSENCE OF the circumstance with which the proposition itself is associated (allowing for the purposes of this discussion the assumption of a naive semantics). But what about the part of the concept of negation having to do with otherness - THE PRESENCE OF any of the many other circumstances different from (viz. refutatory with respect to) the circumstance with which the proposition itself is associated. It is indeed this further dimension of negation, of otherness, which is struggled with by Aristotle and which he attempts to explicate and systematize with the notion of privation, and which is struggled with as well by the predecessors of Ratnakirti and by Ratnakirti himself as they return again and again in their long and patient analyses to the notions of absence and otherness and argue painstakingly for their distinctness.

Let us use first $\mathrm{EL}_{2}$ to try to bring some clarity to this historical perspective. In fact, in $\mathrm{EL}_{2}$ we shall find a systematization of precisely these two major dimensions of the concept of negation. Continuing, in the present context, at the intuitive level used above which assumes a naive semantics, $\mathrm{P}_{c}$ is the proposition associated with circumstances which confirm $\mathrm{P}$ while $\neg \mathrm{P}_{c}$ is the proposition associated with the absence of such circumstances. $\mathrm{P}_{r}$ is the proposition associated with the presence of circumstances which refute $\mathrm{P}$ while $\neg \mathrm{P}_{r}$ is the proposition associated with the absence of circumstances the presence of which would refute $\mathrm{P}$.

Using the more general EL logics $\mathrm{EL}_{n}$ helps as well. For although many logicians who have worked on negation did not consider 'levels of certainty', many did, and in any case introducing nontrivial evidence gradations may serve to further illuminate the texture of logics, like EL, which attempt to treat in a unifying manner both absence and otherness. Who better to reference in this regard than one whose logic involved only absolute certainty and yet whose insightful philosophical writings argue strongly for the need to consider gradations of certainty, viz. George Boole. For example, in the introductory remarks to his chapters on probability in The Laws of Thought he argues that the conduct of science in the real world requires constantly 
working with various levels of certainty, indeed saying at one point [Boole, p. 244]:

With the degree of information we possess concerning the circumstances of an event, [...] our expectation [certainty] of it will vary.

Continuing at the intuitive level we have utilized above and again letting $e$ be any of the evidence values in $\mathrm{E}_{n}, \mathrm{P}_{c}: e$ is the proposition associated with evidence which confirms $\mathrm{P}$ at level $e$ while $\neg \mathrm{P}_{c}: e$ is the proposition associated with the absence of such evidence. $\mathrm{P}_{r}: e$ is the proposition associated with the presence of evidence which refutes $\mathrm{P}$ at level $e$ while $\neg \mathrm{P}_{r}: e$ is the proposition associated with the absence of such evidence.

Two of the aspects of this explication of negation provided by EL which are important to note are the following. First, Aristotle and Ratnakirti, along with many other figures in the history of logic, regularly included in their analyses various theses about relational connections between these two dimensions of negation. For example, in the second passage above from the Prior Analytics we find Aristotle arguing a thesis which in $\mathrm{EL}_{2}$ corresponds to the implication $\mathrm{P}_{r} \rightarrow \neg \mathrm{P}_{c}$, while in the second and third passages above of Ratnakirti we find the thesis that (naively) the meaning of the proposition $\mathrm{P}$ is given by what in $\mathrm{EL}_{2}$ corresponds to the assertion of the conjunction $\mathrm{P}_{c}$ AND $\left(\neg \mathrm{P}_{r}\right)$. Such assertion of various theses relating absence and otherness, it is crucial to note, is totally absent from the basic framework of EL. Rather, in the various directions made clear by examination of the nature of each problem domain to which EL may be applied, theses appropriate to each domain can be axiomatized over EL, and this axiomatized extension of EL will be the logic used within that domain. Second, in EL we have been able to provide the above explication of these two aspects of the history of negation precisely because EL provides a fundamental first-order predicate logic which is adequately equipped for carrying out such explication since it, so to speak, houses under one framework both of these aspects of negation.

\section{Discussion}

Let us briefly indicate how EL may be further used to provide a more detailed analysis of the concept of negation. Let $\mathcal{A}$ be a structure (model) in the semantics for $\mathrm{EL}_{n}$. $\mathcal{A}$ provides interpretations for all function and predicate symbols in $\mathrm{EL}_{n}$, as briefly surveyed in Section 3 above. Readers who are familiar with the formal structure of logic and model theory, or who spend 
some time with [Faust 1982, 1993], may give a precise interpretation to the following discussion. For others, an informal interpretation of the discussion is possible, wherein a structure $\mathcal{A}$ is thought of as providing a 'world' in which meaning can be found for each atomic formula in $\mathrm{EL}_{n}$, in other words in which a complete set of circumstances is given. As before, it suffices here to consider just the propositional fragment of EL.

Note that any structure $\mathcal{A}$ for $\mathrm{EL}_{n}$ provides a certain level of witnessing with respect to a proposition $\mathrm{P}$, namely an evidential pair consisting of an evidence level for the evidence confirming $\mathrm{P}$ and an evidence level for the evidence refuting $\mathrm{P}$. Secondly, $\mathcal{A}$ provides, with the degree of its evidence confirming $\mathrm{P}$ together with its absence of a certain degree of evidence refuting $\mathrm{P}$, a certain level of affirmation of $\mathrm{P}$. And thirdly, $\mathcal{A}$ provides, with its absence of a certain degree of evidence confirming $\mathrm{P}$ together with the degree of its evidence refuting $\mathrm{P}$, a certain level of denial of $\mathrm{P}$. Indeed, the second way of viewing the interpretation $\mathcal{A}$ provides for $\mathrm{P}$ may come close to a reasonable, possibly even satisfactory, realization of the differentiation theory of meaning of Indian logic, wherein, roughly, to assert that something is a cow means it manifests the presence of all aspects of 'cowness' together with the absence of any aspect of 'noncowness'. For, under the second way of viewing any model $\mathcal{A}$, it similarly provides a certain level of confirmation of $\mathrm{P}$ together with an absence of a certain level of evidence refutatory of P. Hence, those models $\mathcal{A}$, which provide the maximum amount of evidence confirmatory of $\mathrm{P}$ together with the absence of even the minimum amount of evidence refutatory of $\mathrm{P}$, might be said to manifest the meaning of $\mathrm{P}$.

It is easy to make all this precise in EL, and we proceed now to do so. We limit ourselves as before to the case of a single proposition symbol P. For ease of expression we make the following notational definitions:

$$
\begin{aligned}
& \mathrm{P}_{e^{\prime}}^{e} \stackrel{\text { def }}{=} \mathrm{P}_{c}: e \text { AND } \mathrm{P}_{r}: e^{\prime}, \\
&{ }^{e} \mathrm{P}^{e^{\prime}} \stackrel{\text { def }}{=} \mathrm{P}_{c}: e \text { AND }\left(\neg \mathrm{P}_{r}: e^{\prime}\right), \\
&{ }_{e} \mathrm{P}_{e^{\prime}} \stackrel{\text { def }}{=}\left(\neg \mathrm{P}_{c}: e\right) \text { AND } \mathrm{P}_{r}: e^{\prime} .
\end{aligned}
$$

First, let us say that $\mathcal{A}\left(e, e^{\prime}\right)$-witnesses $\mathrm{P}$, denoted $\mathcal{A} \models_{e^{\prime}}^{e} \mathrm{P}$, iff

$$
\mathcal{A} \vDash \mathrm{P}_{e^{\prime}}^{e} \quad \text { and } \quad\left(\left(f>e \text { or } f^{\prime}>e^{\prime}\right) \text { implies } \mathcal{A} \not \models \mathrm{P}_{f^{\prime}}^{f}\right) .
$$

Notice that this concept of witnessing formalizes the fact that any model "witnesses a pair of circumstances with regard to the proposition P", providing an evidence level for $\mathrm{P}_{c}$ together with an evidence level for $\mathrm{P}_{r}$. Second, 
let us say that $\mathcal{A}\left(e, e^{\prime}\right)$-affirms $\mathrm{P}$, denoted $\mathcal{A} \vDash^{e, e^{\prime}} \mathrm{P}$, iff

$$
\mathcal{A} \vDash{ }^{e} \mathrm{P}^{e^{\prime}} \text { and }\left(\left(f>e \text { or } f^{\prime}<e^{\prime}\right) \text { implies } \mathcal{A} \not \models{ }^{f} \mathrm{P}^{f^{\prime}}\right) .
$$

Third, let us say that $\mathcal{A}\left(e, e^{\prime}\right)$-denies $\mathrm{P}$, denoted $\mathcal{A} \vDash_{e, e^{\prime}} \mathrm{P}$, iff

$$
\mathcal{A} \vDash{ }_{e} \mathrm{P}_{e^{\prime}} \quad \text { and } \quad\left(\left(f<e \text { or } f^{\prime}>e^{\prime}\right) \text { implies } \mathcal{A} \not \models{ }_{f} \mathrm{P}_{f^{\prime}}\right) .
$$

Note that if $e^{\prime}<1$, then

$$
\mathcal{A} \vDash_{e^{\prime}}^{e} \mathrm{P} \quad \text { iff } \mathcal{A} \vDash^{e, e^{\prime}+\varepsilon} \mathrm{P} .
$$

That is, provided $e^{\prime}<1$ and because of the maximality implied by the second condition in the definition of witnessing, the presence in $\mathcal{A}$ of refutatory evidence with respect to $\mathrm{P}$ at level $e^{\prime}$ is equivalent to the absence in $\mathcal{A}$ of refutatory evidence with respect to $\mathrm{P}$ at level $e^{\prime}+\varepsilon$. And similarly if $e<1$, then

$$
\mathcal{A} \vDash_{e^{\prime}}^{e} \mathrm{P} \quad \text { iff } \quad \mathcal{A} \vDash_{e+\varepsilon, e^{\prime}} \mathrm{P} .
$$

Finally, let us say that $\mathcal{A}$ maximally affirms $\mathrm{P}$ iff $\mathcal{A} \vDash^{1, \varepsilon} \mathrm{P}$, which is equivalent to $\mathcal{A} \vDash_{0}^{1} \mathrm{P}$, while $\mathcal{A}$ maximally denies $\mathrm{P}$ iff $\mathcal{A} \vDash_{\varepsilon, 1} \mathrm{P}$, which is equivalent to $\mathcal{A} \models_{1}^{0} \mathrm{P}$ (where the failure of $\mathcal{A}$ in any case to stipulate any evidence level is indicated here by the evidence level of 0 ). Thus, one might say that it is those models $\mathcal{A}$ which maximally affirm $\mathrm{P}$ in which the meaning of $\mathrm{P}$ is manifested.

We have hereby constructed in $\mathrm{EL}_{n}$ one of the possible explicata related to aspects of the connection between absence and otherness which both Aristotle and Ratnakirti were attempting to uncover and analyze. From Ratnakirti's point of view it is in some sense the case that the semantics (meaning) to be associated with a proposition $\mathrm{P}$ should provide both 'the presence of absolute evidence which is confirmatory of $\mathrm{P}$ ' and 'the absence of any evidence which is refutatory of $\mathrm{P}$ ', which for us is precisely any structure $\mathcal{A}$ such that $\mathcal{A}$ maximally affirms $\mathrm{P}$, that is any structure $\mathcal{A}$ such that $\mathcal{A} \vDash$ $\mathrm{P}_{c}: 1$ AND $\left(\neg \mathrm{P}_{r}: \varepsilon\right)$, or equivalently such that $\mathcal{A}(1, \varepsilon)$-affirms $\mathrm{P}$. This, then, is an example of the type of analysis which is supported by EL and which may help in further elucidating various of the problematical aspects of negation.

In summary, EL has provided a framework within which we have been able to isolate, analyze, and attempt further clarification of, a least two of the aspects of negation which have been grappled with repeatedly throughout the history of logic. Additionally, more may be possible, for as pointed out 
above and in Section 4, a number of the relational intricacies involved in these aspects of negation (and other aspects as well) are expressible in EL, and hence may be subject to further clarification through the analysis of appropriate axiomatizable extensions of EL.

Some small-scale experimentation with EL, in relation to its efficacy in attacking various knowledge representation and knowledge processing problem areas of AI, has been initiated by the writer with a Resolution-based implementation of EL. It is hoped that this paper, in conjunction with its companion papers [Faust 1993, 1994, 1997] will contribute to the broadening of such experimentation. As a result of such experimentation it can be expected that further development of logics such as EL, which are foundational and which are conceptually antecedent to Classical Logic, will occur and will prove helpful in addressing some of the very substantial problem areas of AI.

Finally, it is hoped that our analysis of the concept of negation, which involved both (1) looking at a very real current problem in the science of AI and (2) looking at the earlier work already done in attempting to address this problem, may serve as an example of the fact that many of the most problematic areas of current science indeed involve old problems in new guises. With such a perspective, we can better view problems evolutionarily, gaining helpful input from past ruminations on the problem as well as seeing more clearly how our current contributions to understanding the problem need be but one more intermediate stage in the long history and possibly longer future of work on the problem.

\section{References}

Aristotle, Prior Analytics, The Loeb Classical Library: Harvard University Press, 1967.

Boole, G, An Investigation of the Laws of Thought, Dover Publications.

Carnap, R., Logical Foundations of Probability, University of Chicago Press, 1950.

Dubois, D., and H. Prade, "An Introduction to possibilistic and fuzzy logics", Nonstandard Logics for Automated Reasoning, Academic Press, 1988, 287-326.

Faust, D., "The Boolean algebra of formulas of first-order logic", Annals of Mathematical Logic 23 (1982), 27-53 (MR84f:03007).

Faust, D., "The representation and processing of uncertain information: status report and prospectus for information technology applications", Procedings of the International Conference on Information Technology (ICIT 1990 - Malaysia), E275-E295. 
Faust, D., "Observations on the construction of a foundation for the handling of uncertainty', Proceedings of the Asian Mathematical Conference 1990 (Singapore: World Scientific, 1992), 140-143.

Faust, D., "The concept of evidence" (1993), submitted (preprint available from the author).

Faust, D., "The concept of evidence" (abstract), The Journal of Symbolic Logic 59 (1994), 347-348.

Faust, D., "Evidence and negation" (abstract), The Bulletin of Symbolic Logic 3 (1997), 364.

Matilal, B. K., The Navya-Nyaya Doctrine of Negation, Harvard University Press, 1968.

Russell, B., "Vagueness", Australasian Journal of Philosophy 1 (1923), 84-92.

Sharma, D., The Differentiation Theory of Meaning in Indian Logic, Mouton, 1969.

Tarski, A., Logic, Semantics, and Metamathematics: Papers from 1923 to 1938 , translated by J. H. Woodger, Oxford University Press, 1956.

DON FAUST

Department of Mathematics and Computer Science

Northern Michigan University

Marquette, MI 49855, USA 